\title{
Remoção de hábito de sucção labial com uso de placa lábio ativa: relato de caso
}

\author{
Removal of lip suction habit using a lip bumper: a case report \\ Eliminación del hábito de succión labial utilizando lip bumper: reporte de un caso
}

\begin{abstract}
Resumo
Objetivo: Este trabalho tem como objetivo geral verificar a importância do diagnóstico de maloclusões, implicações clinicas e tratamento precoce por meio da ortodontia preventiva e interceptativa, abordando os benefícios do uso da PLA e realizando a remoção o habito de sucção labial pelo uso da mesma. Metodologia: Partindo da permissão do responsável da paciente por meio do Termo de Consentimento Livre e Esclarecido (TCLE) em partilhar sua imagem para devido fim, o presente estudo trata de um relato de caso clínico de cunho analítico descritivo (Pereira et al., 2018). Relato de Caso: Paciente do sexo feminino, 12 anos de idade, Padrão II por deficiência mandibular e maxilar, com hábito de sucção labial, vestibularização acentuada dos incisivos superiores. A terapia resultou na eliminação do hábito e melhora na inclinação dos incisivos superiores. Evitando tratamentos complexos futuros, dispondo melhoria e qualidade de vida para a paciente. Considerações Finais: Os hábitos bucais deletérios são fatores que levam as maloclusões em pacientes na dentadura mista. A Placa lábio ativa foi o tratamento interceptativo capaz de eliminar o hábito de sucção labial em um curto período de tempo.
\end{abstract}

Palavras-chave: Placa lábio; Má oclusão; Hábito de sucção labial. 


\begin{abstract}
Objective: This study aims to verify the importance of diagnosing malocclusions, clinical implications and early treatment through preventive and interceptive orthodontics, addressing the benefits of using PLA and removing the habit of lip sucking through its use. Methodology: Based on the permission of the patient's guardian through the Informed Consent Form (FICF) to share their image for the proper purpose, this study is a clinical case report of a descriptive analytical nature (Pereira et al., 2018 ). Case Report: Female patient, 12 years old, Pattern II due to mandibular and maxillary deficiency, with lip sucking, accentuated vestibularization of the upper incisors. The therapy resulted in elimination of the habit and improvement in upper incisor inclination. Avoiding future complex treatments, providing improvement and quality of life for the patient. Final Considerations: The deleterious oral habits are factors that lead to malocclusions in patients with mixed dentition. Active lip plaque was the interceptive treatment capable of eliminating the habit of lip sucking in a short period of time.
\end{abstract}

Keywords: Lip plate; Malocclusion; Lip sucking habit.

\title{
Resumen
}

Objetivo: Este estudio tiene como objetivo verificar la importancia del diagnóstico de las maloclusiones, las implicaciones clínicas y el tratamiento temprano a través de la ortodoncia preventiva e interceptiva, abordando los beneficios del uso de PLA y eliminando el hábito de chuparse los labios mediante su uso. Metodología: Con base en el permiso del tutor del paciente a través del Formulario de Consentimiento Informado (FICF) para compartir su imagen con el propósito adecuado, este estudio es un reporte de caso clínico de carácter analítico descriptivo (Pereira et al., 2018). Caso clínico: Paciente de sexo femenino, 12 años, Patrón II por deficiencia mandibular y maxilar, con succión de labios, vestibularización acentuada de los incisivos superiores. La terapia resultó en la eliminación del hábito y la mejora de la inclinación de los incisivos superiores. Evitando futuros tratamientos complejos, aportando mejora y calidad de vida al paciente. Consideraciones finales: Los hábitos bucales deletéreos son factores que conducen a maloclusiones en pacientes con dentición mixta. La placa labial activa fue el tratamiento interceptivo capaz de eliminar el hábito de chuparse los labios en un corto período de tiempo.

Palabras clave: Placa de labios; Maloclusión; Hábito de chuparse los labios.

\section{Introdução}

Defina-se oclusão ideal uma relação entre o equilíbrio das bases osséas, sistema estomatognático e posicionamento correto dos elementos dentários. Quaisquer desvios posturais em funções anormais pela musculatura orofacial resultarão e colaborarão para o desenvolvimento das maloclusões (Almeida et al., 2000). Sendo definido como terceira prioridade na escala de problemas em crianças na fase de dentição decídua ou mista no Brasil (OMS, 2010), desenvolvendo-se assim hábitos bucais deletérios.

Os hábitos deletérios mais abordados na literatura são a respiração bucal, deglutição atípica, uso de chupetas e hábito de sucção labial e digital que podem gerar impactos negativos na qualidade de vida da criança e adolescente por meios de fatores biológicos, psicológicos e sociais. Entre os biológicos ocorrem às alterações no desenvolvimento esquelético, dentoalveolares e neuromusculares. Nos psicológicos, a mudança emocional acarretando as relações sociais como a relação a si mesmo progredindo desde a infância. Diante disso, as funções que partem em potencial no agravamento e desarmonia no padrão normal do crescimento facial, são conceituadas deletérias (Borrie et al.,2015).

A respiração bucal pode acarretar tanto pelos fatores genéticos como pela obstrução na cavidade nasal, assim sofrendo alteração nas vias aéreas, outorgando na simetria facial dos pacientes (Okuro et al., 2011). A sucção labial pode ser subsequente por desordens emocionais, necessidades nutricionais e levando as condições de idade do paciente e sinais clínicos. Envolvendo as mudanças posturais e neurológicas na fase de crescimento, tendo como sintomas a insônia, olheiras, cansaço e apetite reduzido (Seias et al., 1988; Borrie et al., 2015).

O tratamento preventivo e interceptativo busca adotar medidas para oclusão funcional sem intervenções complexas, destarte a importância de diagnósticos precisos (Bruggemann et al., 2013). Trazendo benefícios na evolução dentária e na musculatura dos pacientes, precavendo perdas e danos que interferem nas forças musculares e na assimetria (Serra et al., 1997; Almeida, 2013; Ling et al., 2018). 
A PLA é uma das primeiras indicações nos casos interceptativos, pelas vantagens de baixo custo e aceitação em pacientes pediátricos. Tem como finalidade o ganho de comprimento na arcada dentária, melhoria no declive dos incisivos inferiores e atenuação do overjet (Vanarsdall Jr et al., 2009, Filho et al., 2008; Hashish \& Mostaf 2009; Celentano et al., 2011).

Este trabalho tem como objetivo geral verificar a importância do diagnóstico de maloclusões, implicações clinicas e tratamento precoce por meio da ortodontia preventiva e interceptativa, abordando os benefícios do uso da PLA e realizando a remoção o habito de sucção labial pelo uso da mesma. Evitando tratamentos complexos futuros, dispondo melhoria e qualidade de vida para a paciente.

\section{Metodologia}

Partindo da permissão do responsável da paciente por meio do Termo de Consentimento Livre e Esclarecido (TCLE) em partilhar sua imagem para devido fim, o presente estudo trata de um relato de caso clínico de cunho analítico descritivo (Pereira et al.,2018), realizado na clínica odontológica do Centro Universitário Fametro, na disciplina de estágio Clínica de Atenção Integrada Infantil, do curso de graduação do CEUNI Fametro.

\section{Resultados e Discussão}

Paciente gênero feminino, 12 anos, parda, procurou a Clínica Odontológica Infantil do Centro Universitário Fametro junto com sua responsável, relatando como queixa principal "dentes para frente". Na anamnese foi observado que a paciente havia um ótimo estado de saúde geral e a responsável também relatou que a mesma possuía o habito de morder os lábios.

No exame extraoral observou-se que a paciente é braquifacial com padrão II, perfil convexo, e sem selamento labial passivo, com simetria facial (Figura 1).

Figura 1 - (A, B e C). Fotografias iniciais da face (frontal, sorrindo e perfil).

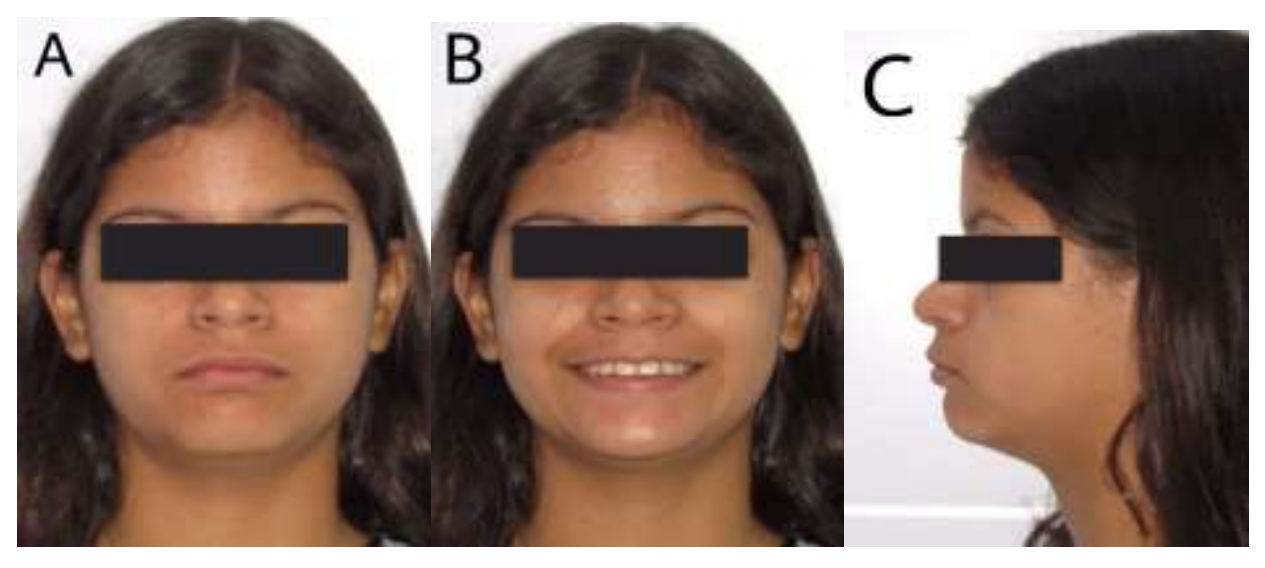

Fonte: CIMO.

No exame Intra-oral, visual e tátil percebeu-se que a paciente estava no fim do período intertransitório da dentadura mista, apresentava relação de molar classe I de Angle no lado direito e classe II de Angle no lado esquerdo. Os incisivos superiores vestibularizados, os incisivos inferiores lingualizados e presença de Tórus mandibular (Figura 2). 
Figura 2 - A: fotografias iniciais intraorais frontal; B: fotografias iniciais intraorais lateral esquerda; C: fotografias iniciais intraorais lateral direita; D: fotografias iniciais intraorais oclusal inferior; E: fotografias iniciais intraoral superior (Fonte: CIMO).

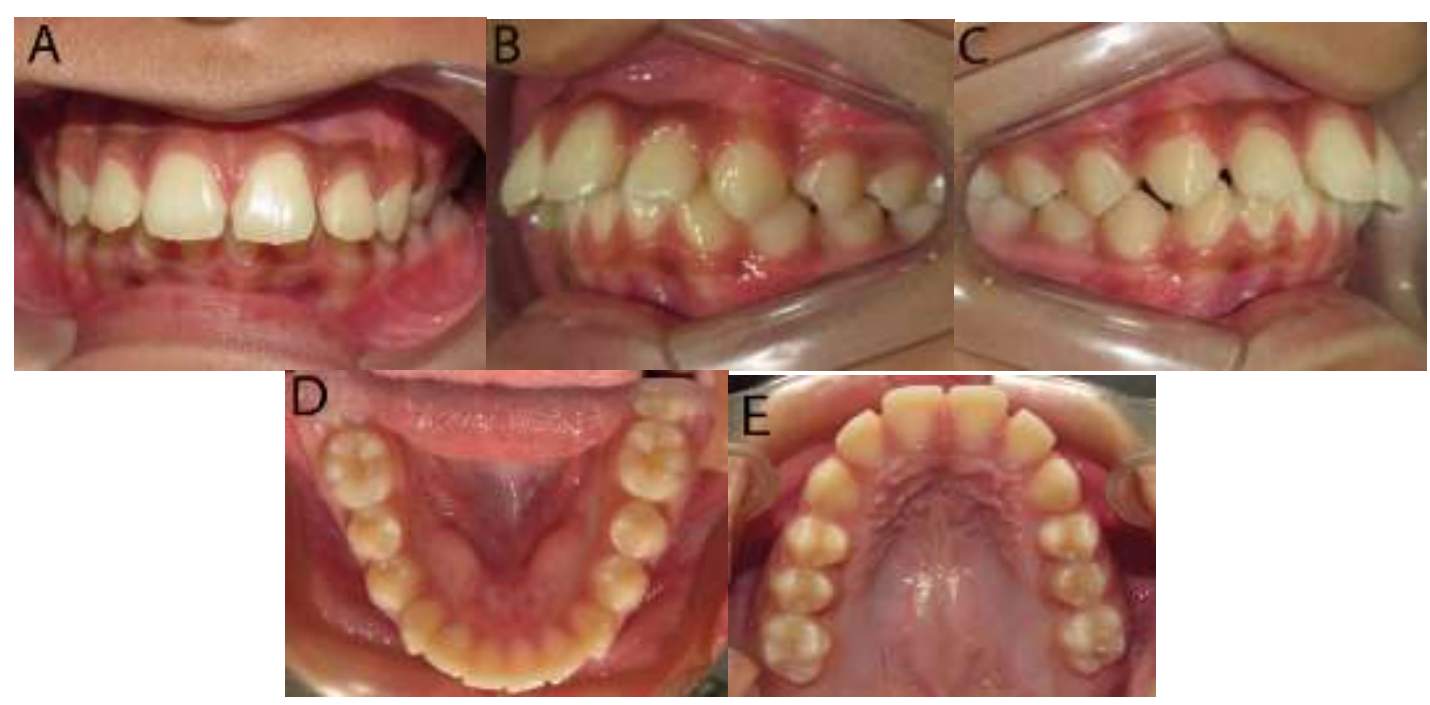

Fonte: CIMO.

Foi solicitado a documentação básica, e traçado USP para melhor analisar o caso da paciente. Na análise da radiografia panorâmica, verificou-se a presença dos elementos 17, 27, 37 e 47 em desenvolvimento. De acordo com a telerradiografia e análise cefalométrica USP, o diagnóstico apresentado: Protusão maxilar e protusão mandibular, Classe II esquelética, incisivos superiores e inferiores vestibularizados e inclinados para vestibular (Figura 3; Quadro 1).

Figura 3 - A) Radiografia panorâmica; B) Traçado cefalométrico análise USP.
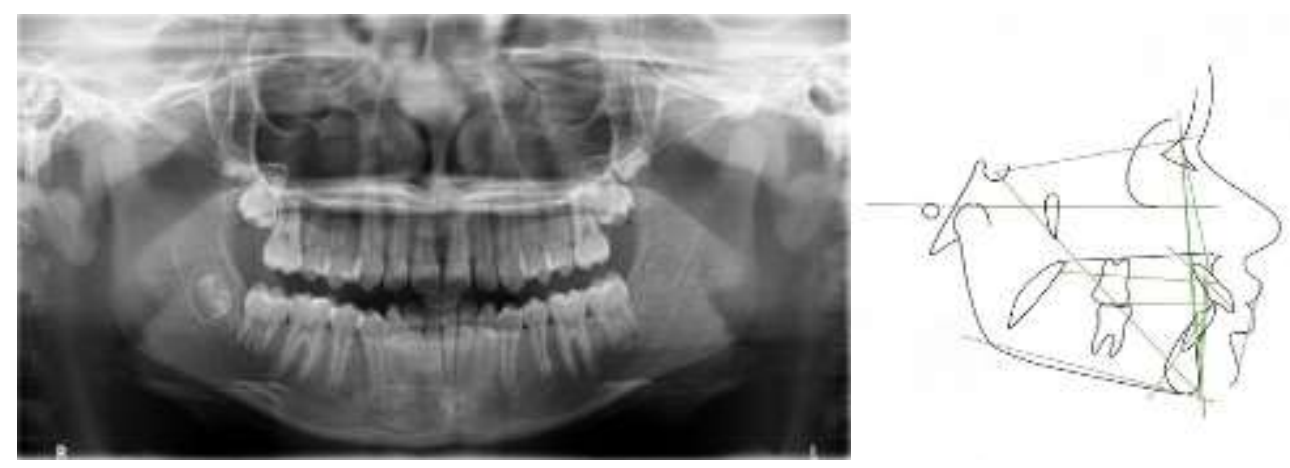

Fonte: CIMO. 
Quadro 1 - valores Cefalométricos padrão e iniciais (análise USP)

\begin{tabular}{|l|l|l|}
\hline & Padrão & Valores iniciais \\
\hline SNA & $82^{\circ}$ & $89^{\circ}$ \\
\hline SNB & $80^{\circ}$ & $82^{\circ}$ \\
\hline ANB & $2^{\circ}$ & $7^{\circ}$ \\
\hline FMA & $25^{\circ}$ & $16^{\circ}$ \\
\hline 1 -NA & $4 \mathrm{~mm}$ & $5,28 \mathrm{~mm}$ \\
\hline $1 . \mathrm{NA}$ & $22^{\circ}$ & $30^{\circ}$ \\
\hline $1 . \mathrm{NB}$ & $25^{\circ}$ & $32^{\circ}$ \\
\hline $1-\mathrm{NB}$ & $4 \mathrm{~mm}$ & $6,10 \mathrm{~mm}$ \\
\hline H.NB & $101,5^{\circ}$ & 16 \\
\hline (S-N). (Go-Me) & $32^{\circ}$ & $27^{\circ}$ \\
\hline IMPA & $87^{\circ}$ & $103^{\circ}$ \\
\hline
\end{tabular}

Fonte: CIMO

Inicialmente foram instaladas as ligas separadoras ortodônticas nas faces mesiais dos elementos 36 e 46 . Uma semana depois foi realizada a cimentação das bandas ortodônticas nos primeiros molares inferiores permanentes com ionômero de cimentação (Ionomaster C) (Figura 4).

Figura 4 - A) Ligas separadoras ortodônticas; B) Bandas cimentadas com tubos para PLA; C) PLA instalada.

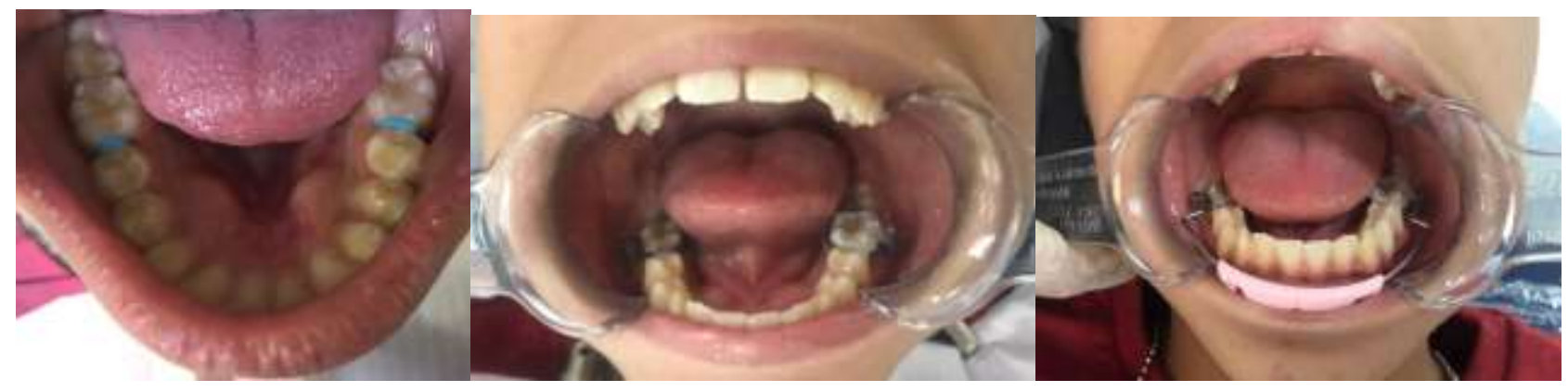

Fonte: Autores.

Nas consultas seguintes a PLA foi removida e ativada no sentido antero posterior de forma que ficasse afastada $5 \mathrm{~mm}$ do lábio inferior assim impedindo o contato dos tecidos moles com os dentes inferiores e a profilaxia realizada na paciente

Após 2 meses com a PLA foi relatado pela responsável que a paciente não realizava mais o hábito de sucção labial e observou-se uma melhora no selamento labial pela reanálise facial e na estética do sorriso (Figura 5) 
Figuras 5 - (A, B e C). Fotografias da face (frontal, perfil e sorindo).

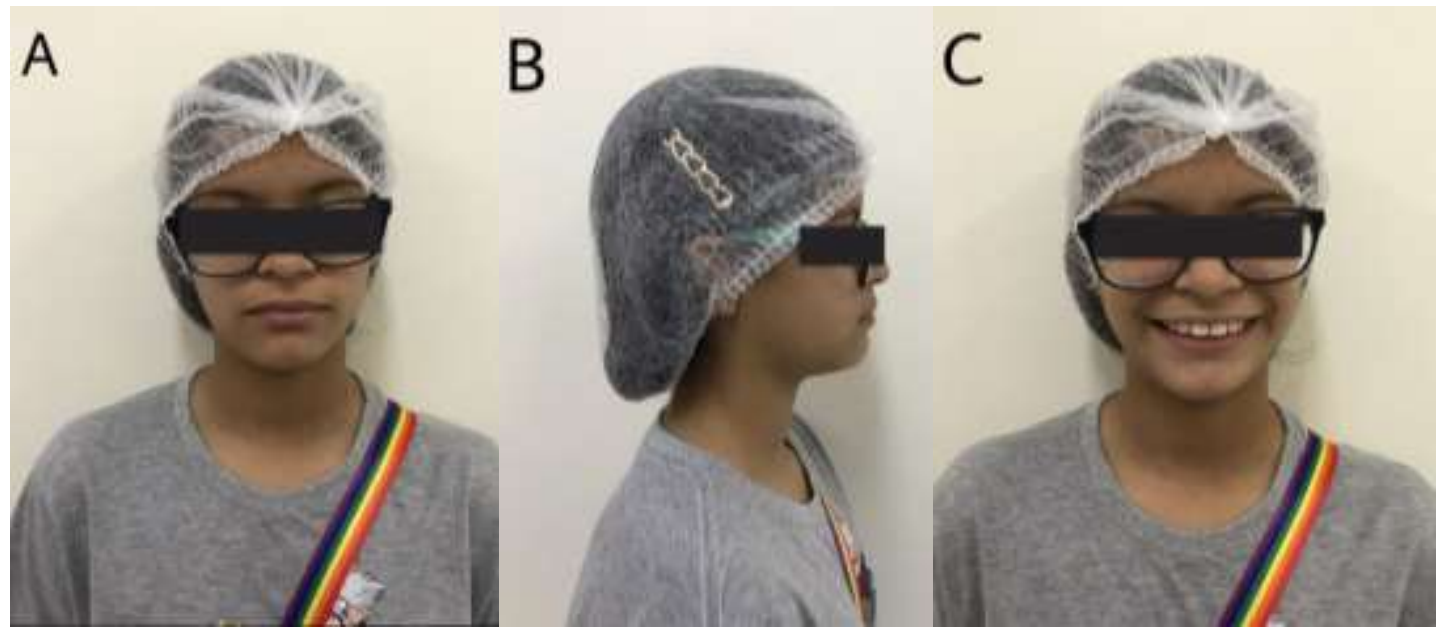

Fonte: Autores.

\section{Discussão}

Segundo Padmapriya (2018) uma má oclusão está literalmente relacionada a posição dos elementos dentários, podendo ser diagnosticada ainda na dentura mista onde envolve periocidamente os sextantes anteriores superiores.

Os hábitos bucais deletérios têm sido associados as mal oclusões em crianças (Pizzol et al., 2004; Faltin et al., 2011; Ling et al., 2018), portanto a ortodontia interceptativa tem como objetivo equilibrar e estimular o desenvolvimento correto da oclusão e das estruturas faciais (Santana et al., 2020). Assim, no presente caso optou-se pela remoção do hábito de interposição labial com uso do aparelho interceptativo placa lábio ativa.

Dessa forma os dados constataram-se com a literatura, qual o hábito de suçẽa labial ocasiona a protusão dentária superior gerando um grande transtorno na fase da dentição mista, assim impossibilitando o selamento labial e resultando em alterações nos músculos e ossos (Amin et al., 2010). Knorst (2019) afirma que as maloclusões agravam na qualidade de vida em crianças e adolescentes, qual a vergonha de sorrir e falar estão presentes na rotina dos mesmos. Estes dados estão de acordo com o presente relato com a literatura, onde a responsável relatou o desconforto da criança em sorrir.

A Placa Lábio Ativa ou "Lip Bumper" é um aparelho removível, multifuncional, que, de acordo com Almeida et al., (2006), repassa as forças criadas pela musculatura aos dentes de ancoragem, deixando a ação dessas forças nos dentes anteriores e, ao mesmo tempo, liberando uma maior ação na língua. Em 1979 Graber sugeriu o uso da PLA para eliminação do hábito de sucção labial, qual tem como função o afastamento do tecido mole e dos músculos, dessa forma reduzindo a pressão dos lábios e das bochechas sobre os elementos dentários. Ele consiste conforme Janson et al., (2013), em um arco labial de aço inoxidável e um escudo, geralmente de acrílico, entretanto pode ser de plástico, ou feito pelo próprio fio coberto por uma proteção de plástico, o aparelho contorna externamente o perímetro do arco dentário inferior. De acordo com Ferreira (2008), as extremidades do fio do arco são introduzidas, de preferência, nos tubos vestibulares das bandas. Conforme Maahs (2005) em pacientes não colaboradores pode-se amarrar o aparelho aos tubos com fio de amarrilho. Ja Almeida (2012) indica o uso por um intervalo de tempo diário máximo, sendo removível para a higiene, alimentação e prática de esportes físicos. A PLA foi instalada e adaptada com 3mm de distância dos incisivos inferiores de forma removível, facilitando a alimentação e higienização. Durante o acompanhamento o aparelho era removido, ativado e a profilaxia realizada na paciente.

Janeth (2017) em um caso clínico atestou que a PLA foi uma alternativa eficaz, eliminando o hábito de suçã̃o labial e a interposição labial em dentadura mista. Tendo como aumento do perímetro do arco dentário em muitos casos (Celetano et al., 2011), ocorrendo a redução do apinhamento ântero-inferior, por entre a vestibularização dos incisivos inferiores (Almeida, 2012), onde a pressão da musculatura labial é reduzida, tendo como efeito uma maior ação na língua sobre os dentes (Germeç 
\& Taner 2008). Hodge afirma que para eliminação do hábito é indicado o aparelho de forma adaptada que o escudo fique afastado dos incisivos inferior e na posição mais superior (Hodge et al., 1997).

A condição do prazo de tratamento ainda é debatida nos estudos. Germeç e Taner (2005) relataram o uso efetivo de 3 meses de tratamento. Diferente em relação a 1 ano de tratamento frente estudos de Santana et al., (2020) e Vanarsdall Jr et al., (2009). No presente estudo foram necessários 2 meses e meio de tratamento com a placa lábio ativa para a remoção do hábito em base de Germeç \& Taner (2005). A forma como a placa lábio ativa mudo o equilíbrio entre as forças lingual e vestibular nos dentes esclarece o resultado deste estudo. Com o tempo a dentição adapta-se a um novo sistema de forças, agora produzido pela placa lábio ativa, e restabelece o equilíbrio; Destarte, não há mais alteração no comprimento do arco (Murphy et al., 2003).

A paciente encontra-se em acompanhamento, segundo o relato da responsável a mesma não possui mais vergonha ao sorrir e sente-se mais feliz após tratamento. Clinicamente sucedeu melhora na vestibularização dos incisivos superiores, sendo assim o aparelho interceptativo foi capaz de atingir a finalidade proposta para o caso clínico.

A paciente entrará no tratamento com disjuntor Hyrax como continuação da terapia ortopédica e ortodôntica.

\section{Considerações Finais}

Os hábitos bucais deletérios são fatores que levam as mal oclusões em pacientes na dentadura mista. A Placa lábio ativa foi o tratamento interceptativo capaz de eliminar o hábito de sucção labial em um curto período de tempo. Outros benefícios foram observados como diminuição da vestíbulo versão dos incisivos superiores já que o aparelho restringiu as forças periorais sobre os dentes.

\section{Referências}

Almeida, R. R., et al. (2000). Etiologia das más oclusões - causas hereditárias e congênitas, adquiridas gerais, locais e proximais (hábitos bucais). Rev. Dent. Press Ortodon. Ortop. Facial. 5(6),107-129.

Almeida, F. L., Silva, A. M. T., \& Serpa, E.O. (2009). Relação entre má oclusão e hábitos em respiradores orais. Revista Cefac, $11,86$.

Bernabé, E., Sheiham, A., \& Oliveira, C. M. (2008). Condition-specific impacts on quality of life attributed to malocclusion by adolescents with normal occlusion and class I, II and III malocclusion. Angle Orthod, 78(6), 977-982.

Borrie, F. R. P., et al. (2015). Interventions for the cessation of nonnutrive sucking habits in children (Review). Cochrane Database Syst. Rev., 3, 10.1002/14651858.CD008694.pub2.

Celentano, G., Longobardi, A., Cannavale, R., \& Perillo, L. (2011). Mandibular lip bumper for molar torque control. Prog Orthod, 12(1), 90-9.

Da Silva, J. M. D., et al. (2020). Prevalência de maloclusões em crianças escolares e sua associação com hábitos bucais deletérios. Diversitas Journal, 5(3), 1818-1827.

Ferreira, F. V. (2008). Ortodontia: diagnóstico e planejamento. Artes Médicas.

Graber, T. M. (1979). The use of muscle forces by simple orthodontic appliances. Am J Orthod, 76(1), 1-20.

Germeç, D., Taner, T. U. (2005). Lower lip sucking habit treated with a lip bumper appliance. Angle Orthod, 75(6), 1071-1076.

Gomes, E. R. L., et al. (2021). Postura e carga de peso podal em crianças com o uso de aparelhos ortodônticos ou ortopédicos funcionais dos maxilares: um estudo longitudinal. Brazilian Journal of Development, 7(3), 26644-26655.

Janeth, C. L. G. (2017). El Lip Bumper como tratamiento interceptivo del hábito de interposición labial [Monografia]. Guayaquil (EC) - Universidad de Guayaquil.

Knors, J. K., Menegazzo, G. R., Emmanuelli, B., Mendes, F. M., \& Ardenghi, T. M. (2019). Effect of neighborhood and individual social capital in early childhood on oral health-related quality of life: a 7-year cohort study. Qual Life Res, 28(7), 1773-1782.

Ling, H. T., Sum, F. H., Zhang, L., Yeung, C. P., Li, K. Y., Wong, H. M., et al. (2018). The association between nutritive, nonnutritive sucking habits and primary dental occlusion. BMC Oral Health. 18, 145.

Maroneze, M. C., Ardenghi, D. M., Brondani, M., Unfer, B., \& Ardenghi, M. (2019). Dental treatment improves oral health-related quality of life of adolescents: A mixed methods approach. Int J Pediatr Dent, 1-10. 
Research, Society and Development, v. 10, n. 16, e396101624171, 2021

(CC BY 4.0) | ISSN 2525-3409 | DOI: http://dx.doi.org/10.33448/rsd-v10i16.24171

Murphy, C. C., Magness, B. W., English, J. D., Frazier-Bowers, A. S., \& Salas, A. M. (2003). A longitudinal study of incremental expansion using a mandibular lip bumper. Angle Orthod, 73(4), 396-400.

Okuro, R. T., et al. (2011). Respiração bucal e anteriorização da cabeça: efeitos na biomecânica respiratória e na capacidade de exercício em crianças. J. Bras. Pneumol., 37(4), 471-479.

Pereira, C. M., \& Demito, C. F. (2008). Placa Lábio Ativa, Maringá: Dental Press, 7 (Série aparelhos ortodônticos)

Pereira, A. S., Shitsuka, D. M., Parreira, F. J., \& Shitsuka, R. (2018). Metodologia da pesquisa científica. Santa Maria, RS: UFSM, NTE.

Pizzol, K. E. D. C., Bausells, J., Marcomini, E. M. S., Cayetano, M. H., Cauhi L. P., \& Melo R. B. (2004). Tratamento de deglutição com pressão atípica do lábio com placa lábioativa reversa - relato de caso clínico. J Bras Ortodon Ortop Facial, 9(51), 211-7.

Ramos dos Santos, N., Carlos Carvalho Cerqueira, R.., Rocha Soledade, K., \& Rolim Borges-Paluch, L. (2020). Ocorrência de má oclusão em pacientes pediátricos atendidos em uma clínica integrada de odontologia. Textura, 14(1), 50-64, 6.

Santana, L. G., França, E. C., Flores-Mirm, C., Abreu, L. G., Marquesm, L. S., \& Martins-Junior, P. A. (2020). Effects of lip bumper therapy on the mandibular arch dimensions of children and adolescents: A systematic review. Am J Orthod Dentofacial Orthop. 157, 454-65.

Seixas, C. A. O., Almeida, E. F., \& Fattori, L. (2020). Diagnóstico, prevenção e tratamento precoce para hábitos bucais deletérios. J. Bras. Ortod. Ortop. Maxilar, 3(14), 53-60. Odontologia. Textura, 14(1), 50-64.

Vanarsdall-Júnior, R. L., Secchi, A. G., Chung, C., \& Katz, S. H. (2004). Mandibular basal structure response to Lip Bumper treatment in the transverse dimension. Angle Orthod, 74(4), 473-479. 